\title{
Ceniza de cascarilla de arroz como fuente de sílice en sistemas cementicios de ceniza volante y escoria activados alcalinamente
}

\section{Rice husk ash as a source of silica in alkali-activated fly ash and granulated blast furnace slag systems}

\author{
J.M. Mejía(*), R. Mejía de Gutiérrez ${ }^{(*)}$, F. Puertas ${ }^{(*)}$
}

Recepción / Received: 28-IX-12

Aceptación / Accepted: 14-III-13

\section{RESUMEN}

Este estudio evalúa la viabilidad de utilizar un subproducto agroindustrial, la ceniza de cascarilla de arroz (RHA) proveniente de un equipo combustor de una empresa Arrocera en Colombia, como reemplazo total de la sílice aportada por el silicato de sodio comercial en sistemas cementicios activados alcalinamente. Se prepararon pastas de ceniza volante (FA), de escoria de alto horno (GBFS) y un sistema binario 50FA:50GBFS, que fueron activadas por una mezcla de silicato de sodio e hidróxido de sodio, y por dos tipos de RHA. Las mezclas se caracterizaron mecánica, mineralógica y microestructuralmente. Los resultados demuestran que es posible obtener materiales activados alcalinamente con resistencias mecánicas del orden de $42 \mathrm{MPa}$, a 7 días de curado, utilizando el subproducto agroindustrial. Este estudio corrobora que tanto la sílice amorfa como parte de la sílice cristalina presente en RHA tienen la posibilidad de participar en el proceso de activación alcalina, siempre y cuando las condiciones de alcalinidad estén adecuadamente ajustadas.

Palabras clave: cementos alcalinos; ceniza de cascarilla de arroz; waterglass; resistencias mecánicas; microestructura.

\begin{abstract}
This study assesses the viability of using an agro-industrial by-product, rice husk ash (RHA) from a Colombian rice company's combustion facility, as a total replacement for the commercial sodium silicate ordinarily used in alkaliactivated binders. Fly ash (FA), granulated blast furnace slag (GBFS) and binary 50FA:50GBFS blended pastes were activated with a mix of sodium hydroxide and either sodium silicate or one of two types of RHA. The pastes were characterised for strength, mineralogy and microstructure. The findings showed that the agro-industrial by-product can be used to yield alkali-activated materials with 7-day mechanical strengths on the order of $42 \mathrm{MPa}$. The study confirmed that both amorphous silica and part of the crystalline silica present in RHA participate in the alkaline activation process, providing the alkalinity is suitably adjusted.
\end{abstract}

Keywords: alkaline cements; rice husk ash; waterglass; mechanical strength; microstructure.

\footnotetext{
(*) Universidad del Valle (Cali, Colombia).

(**) Instituto de Ciencias de la Construcción Eduardo Torroja (IETcc-CSIC) (Madrid, España).
} 


\section{INTRODUCCIÓN}

La activación alcalina es una técnica que tuvo su origen en el año 1939, los pioneros en el tema fueron A. O Purdon, V. Glukhovsky y J. Davidovits (1-3). Este proceso requiere mínimo de dos componentes básicos, la materia prima de tipo aluminosilicato preferiblemente de naturaleza amorfa o vítrea, y un compuesto alcalino $(4,5)$. El aluminosilicato puede ser de tipo natural como el metacaolín (MK), el cual proviene de un tratamiento térmico de una arcilla caolinítica, o subproductos industriales como la ceniza volante, la escoria siderúrgica de alto horno, lodos de aguas residuales, cenizas de bagazo de caña, entre otros. El mecanismo propuesto, para el fraguado y posterior endurecimiento del geopolimero, consiste de una disolución, un transporte u orientación y una policondensación (5-7). En la primera etapa se da la disolución de las especies aluminosilicato debido a la presencia del medio alcalino (grupos $\mathrm{OH}^{-}$). Posteriormente se da la difusión o transporte de los iones Al y Si disueltos desde la superficie de las partículas hacia el interior de las mismas dando lugar a una fase tipo gel. La policondensación o polimerización de las especies da origen a unas cadenas rígidas o un conjunto de tetraedros enlazados por oxigeno con una estructura que va de amorfo a semicristalino según el caso. Este arreglo deberá contener suficientes cationes alcalinos para balancear el aluminio presente en la estructura con número de coordinación 4 . El producto de la reacción dependiendo del contenido en calcio de la materia prima puede ser de tipo aluminosilicato alcalino hidratado (N-A-S-H) proveniente de compuestos con contenido de calcio reducido (8), o un gel con estructura Dreierketten, como el gel silicoaluminato cálcico hidratado (C-A-S-H), con contenido apreciable de calcio (8-12).

Los productos obtenidos en la activación alcalina son materiales inorgánicos no combustibles, se forman a bajas temperaturas (inferiores a $200{ }^{\circ} \mathrm{C}$ ), alcanzan resistencias mecánicas a corto plazo, son resistentes al fuego y a los ataques ácidos, demostrando el amplio potencial de aplicación de estos materiales en la ingeniería $(5,13,14)$. Estos materiales han tenido un amplio desarrollo y han atraído gradualmente la atención del mundo científico e industrial, al ser considerados materiales revolucionarios, en parte debido a sus ventajas técnicas y ambientales, a la facilidad de implementación de nuevas tecnologías para su producción y a la versatilidad de los diferentes campos de aplicación; entre los cuales se señalan la producción de materiales cementantes de alto desempeño, composites para la industria automovilística y aeronáutica, encapsulamiento de residuos tóxicos y radioactivos, producción de materiales refractarios y aislantes, rehabilitación estructural, entre otros $(5,15,16)$.

Por sus propiedades y menor impacto ambiental en su producción, los materiales activados alcalinamente se

\section{INTRODUCTION}

Alkaline activation was pioneered by A.O. Purdon, V. Glukhovsky and J. Davidovits as long ago as 1939 (1-3). The process calls for at least two basic components, an aluminosilicate raw material (preferably amorphous or vitreous) and an alkaline compound $(4,5)$. The aluminosilicate may be natural, such as the metakaolin (MK) obtained by burning kaolinitic clay, or an industrial by-product such as fly ash, blast furnace slag, water treatment plant sludge or sugar cane bagasse. The mechanism proposed for geopolymer setting and subsequent hardening comprises dissolution, transportation or orientation and polycondensation, in that order (5-7). In the first stage, aluminosilicate species dissolution is induced by the presence of the alkaline medium (OH groups). That is followed by dissolved Al or Si ion diffusion or transportation from the particle surface inward, giving rise to a gel-like phase. Species polycondensation or polymerisation then gives rise to rigid chains or a series of intertwined tetrahedra joined by oxygen atoms with a structure that may range from amorphous to semi-crystalline. This arrangement calls for a sufficient number of alkaline cations to offset the tetra-coordinated aluminium present in the structure. Depending on the calcium content in the raw material, the reaction product may be a sodium aluminosilicate hydrate (N-A-S-H) (compounds with low calcium content (8)) or a dreierkette-based gel such as calcium aluminosilicate hydrate (C-A-S-H) (compounds with a fairly high calcium content) (8-12).

The products of alkaline activation are non-combustible, acid-resistant inorganic materials formed at low temperatures (under $200^{\circ} \mathrm{C}$ ) and characterised by high early age mechanical strength, properties that attest to their potential in a wide range of industrial applications $(5,13,14)$. These well-developed materials have gradually attracted the attention of the scientific and industrial communities, where they are regarded as revolutionary, due to the technical and environmental benefits afforded, the ease with which the new technologies required for their production can be implemented and their versatile applicability. They are used in the production of high performance binders, composites for the automobile and aeronautics industries, toxic and radioactive waste encapsulation, production of fire-proof and insulating materials and structural rehabilitation, among others $(5,15,16)$.

In light of these properties and since alkaline-activated material production is less energy-intensive and emits 
han considerado una opción alternativa al cemento portland para contribuir en la reducción del consumo energético y de las emisiones de gases de efecto invernadero como el $\mathrm{CO}_{2}$ generadas en el proceso de producción del cemento (17-19).

Los compuestos más utilizados como fuente complementaria de sílice en este tipo de sistemas activados alcalinamente son el grupo de los silicatos sódicos y potásicos hidratados y en cuanto al compuesto alcalino generador del $\mathrm{pH}$ se han trabajado con hidróxidos alcalinos fuertes como el $\mathrm{NaOH}, \mathrm{KOH}$ o sales como el $\mathrm{Na}_{2} \mathrm{SO}_{4}$ (5, 2026). Estos productos, en general, son de tipo industrial; particularmente en lo que se refiere al silicato de sodio este se fabrica a partir de materias primas de tipo natural y altas temperaturas $\left(1.300^{\circ} \mathrm{C}\right)$, por lo tanto su producción exige un elevado consumo energético y por otro lado genera la erosión de los terrenos debido a la explotación de las minas para obtener la materia prima elemental que es el óxido de silicio.

En este sentido, el presente trabajo evalúa la viabilidad de utilizar un subproducto agrícola industrial como es la ceniza de cascarilla de arroz producida en un combustor de una empresa Arrocera Colombiana como fuente de sílice en remplazo total del silicato de sodio en sistemas activados alcalinamente de ceniza volante, escoria siderúrgica de alto horno y en un sistema hibrido de ceniza volante y escoria siderúrgica de alto horno. Lo anterior se plantea como una opción tanto económica como ecológica en el proceso de producción de este tipo de materiales activados a la par que contribuye a la valorización del subproducto.

\section{PROCEDIMIENTO EXPERIMENTAL}

\subsection{Materiales}

Los materiales seleccionados para ser activados alcalinamente fueron: ceniza volante (FA) y escoria vítrea de horno alto (GBFS). La primera proviene de una termoeléctrica y la segunda de una siderúrgica en España. La composición química de ambos materiales se muestra en la Tabla 1. En esta Tabla se aprecia que la ceniza es un tipo F de acuerdo con la Norma ASTM C618 y la escoria presenta un coeficiente de basicidad $((\mathrm{CaO}+\mathrm{MgO}) /$ $\left.\left(\mathrm{SiO}_{2}+\mathrm{Al}_{2} \mathrm{O}_{3}\right)\right)$ de 0,92 .

Como fuentes de sílice se utilizó un silicato de sodio comercial con composición química $\mathrm{SiO}_{2} 32,4 \%, \mathrm{Na}_{2} \mathrm{O}$ $13,95 \%$ y $\mathrm{H}_{2} \mathrm{O} 54 \%$ (en peso) y dos clases de ceniza de cascarilla de arroz (RHA). La primera (RHA1) fue obtenida mediante un proceso controlado en el laboratorio, a $600{ }^{\circ} \mathrm{C}$ durante 3 horas. La segunda ceniza (RHA2) fue proporcionada por una empresa Arrocera Colombiana, less greenhouse gas and $\mathrm{CO}_{2}$ than ordinary cement manufacture, they are regarded as a more environmentally sustainable alternative to portland cement (17-19).

The most frequent supplementary sources of silica in these alkali-activated systems are sodium and potassium silicate hydrates, while strong alkalis such as $\mathrm{NaOH}, \mathrm{KOH}$ or salts such as $\mathrm{Na}_{2} \mathrm{SO}_{4}$ have been used to generate high $\mathrm{pH}$ values (5, 20-26). As a rule, these are industrial products. Sodium silicate, for instance, is manufactured from natural raw materials at high temperatures $\left(1300{ }^{\circ} \mathrm{C}\right)$. Its production is therefore energy-intensive; moreover, mining the main prime material, silicon oxide, induces erosion.

The present study therefore assessed the viability of using an agro-industrial by-product, rice husk ash produced in the combustion facility at a Colombian rice processing plant, as a source of silica to fully replace sodium silicate in three systems: alkali-activated fly ash, blast furnace slag and a fly ash-blast furnace slag hybrid. The objective was to enhance the economics and lessen the environmental impact of the manufacture of this type of activated materials, while contributing to the valorisation of an industrial by-product.

\section{EXPERIMENTAL}

\subsection{Materials}

The materials chosen for alkaline activation were fly ash (FA) and granulated blast furnace slag (GBFS). The former was furnished by a coal-fired steam power plant and the latter by a steel mill, both in Spain. The chemical composition of these two materials is given in Table 1. As the table shows, the fly ash conformed to ASTM C618 type $F$, while the slag exhibited a basicity coefficient $\left((\mathrm{CaO}+\mathrm{MgO}) /\left(\mathrm{SiO}_{2}+\mathrm{Al}_{2} \mathrm{O}_{3}\right)\right)$ of 0.92 .

The silica sources used included a commercial sodium silicate with a chemical composition of 32.4 wt\% $\mathrm{SiO}_{2}$, $13.95 w t \% \mathrm{Na}_{2} \mathrm{O}$ and 54 wt\% $\mathrm{H}_{2} \mathrm{O}$ and two types of rice husk ash (RHA). The first (RHA1) was obtained by laboratory-controlled incineration at $600{ }^{\circ} \mathrm{C}$ for 3 hours. The second (RHA2) was furnished by a Colombian rice processor whose combustion facility generated the ash 
siendo un subproducto generado en un equipo combustor en el cual se utiliza la cascarilla de arroz como biomasa para generar energía, que a su vez es utilizada en el proceso de secado del arroz. Cabe anotar que RHA1 y RHA2 tienen un contenido de $\mathrm{SiO}_{2}$ total superior al $90 \%$, pero difieren en su contenido de $\mathrm{SiO}_{2}$ amorfa, tal como se aprecia en la Tabla 1. El contenido de sílice amorfa se realizó acorde al método propuesto por Metha (27) denominado Índice de Sílice Activa, el cual consiste en medir la proporción de sílice disuelta al poner en contacto el material con una solución de $\mathrm{NaOH}$ 0,5 N durante 3 minutos a temperatura de ebullición. En cuanto al reactivo alcalino se utilizó hidróxido de sodio grado analítico (99\%) marca MERCK. as a by-product of husk combustion, the source of energy used to dry the rice. While both RHA1 and RHA2 consisted of over $90 \% \mathrm{SiO}_{2}$, their amorphous $\mathrm{SiO}_{2}$ content differed, as Table 1 shows. The amorphous silica content was found with the active silica index method proposed by Metha (27), which consists of measuring the proportion of silica that dissolves when the material is immersed for 3 minutes in a boiling $0.5 \mathrm{~N} \mathrm{NaOH}$ solution. The alkaline reagent used was MERCK analytical grade (99\%) sodium hydroxide.

Tabla 1 / Table 1

Composición química

Chemical composition

\begin{tabular}{|c|c|c|c|c|}
\hline Compuestos (\%) / Compound (\%) & FA & GBFS & RHA1 & RHA2 \\
\hline $\mathrm{SiO}_{2}$ & 54.4 & 35.3 & 94.4 & 94.1 \\
\hline $\mathrm{Al}_{2} \mathrm{O}_{3}$ & 26.4 & 13.7 & 0.2 & 0.2 \\
\hline $\mathrm{Fe}_{2} \mathrm{O}_{3}$ & 7.0 & 0.4 & 0.2 & 0.2 \\
\hline $\mathrm{MgO}$ & 1.8 & 4.1 & - & - \\
\hline $\mathrm{CaO}$ & 3.2 & 41.0 & - & - \\
\hline $\mathrm{Na}_{2} \mathrm{O}$ & 0.6 & 0.0 & - & - \\
\hline $\mathrm{S}_{2^{-}}$ & - & 1.91 & - & - \\
\hline $\mathrm{SO}_{3^{-}}$ & 0.01 & 0.06 & - & - \\
\hline $\mathrm{SiO}_{2}$ amorfa / amorphous & 45.1 & - & 94.7 & 27.7 \\
\hline LOI & 2.2 & 2.7 & 2.8 & 3.5 \\
\hline $\begin{array}{c}\text { Tamaño medio de partícula }(\mu \mathrm{m}) / \\
\text { Average Particle Size }(\mu \mathrm{m})\end{array}$ & 40.4 & 17.8 & 36.9 & 39.5 \\
\hline
\end{tabular}

La caracterización de FA y de GBFS se recoge en trabajos previos $(28,29)$. La Figura 1 muestra los difractogramas de ambas cenizas de cascarilla de arroz. En ellos se observa la naturaleza amorfa de la ceniza RHA1 y de manera cualitativa se corrobora que RHA2 es de naturaleza más cristalina representada por la presencia de cuarzo (Q) y cristobalita (C).
$F A$ and GBFS were characterised in prior studies $(28,29)$. The diffractograms for the two types of rice husk ash are shown in Figure 1. According to these XRD patterns, ash RHA1 was more amorphous (94\% of total silica content) while RHA2, which contained quartz (Q) and cristobalite (C), was more crystalline (27\% of the total silica was amorphous).

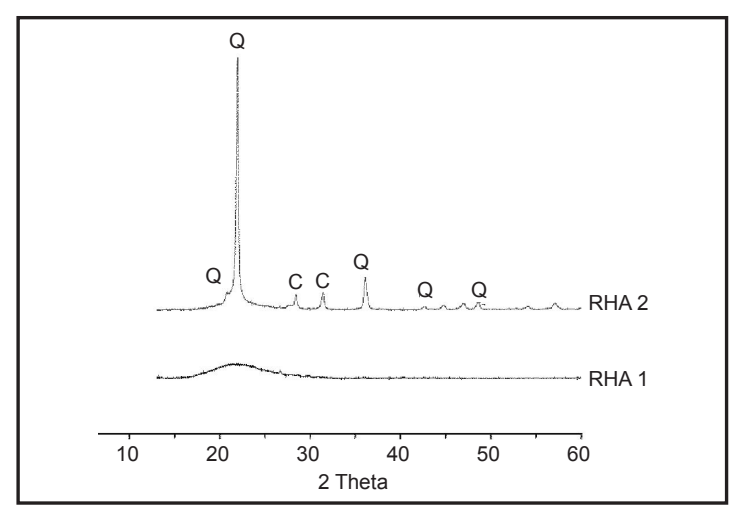

Figura 1. Espectros DRX de RHA1 y RHA2.

Figure 1. RHA1 and RHA2 XRD spectra. 


\subsection{Sistemas preparados y condiciones de procesado}

Se utilizaron tres disoluciones alcalinas preparadas a partir de la mezcla de hidróxido de sodio con silicato de sodio (SS) o ceniza de cascarilla de arroz (RHA1 y RHA2), identificadas como M1, M2 y M3 respectivamente, tal como se muestra en la Tabla 2. Las soluciones M2 y M3 se prepararon con un 1 día de antelación a la aplicación, y se dejaron reposar a temperatura ambiente en recipientes cerrados. Cabe aclarar que cada ceniza de cascarilla de arroz cuenta con un porcentaje distinto de sílice amorfa, RHA1 contiene un $94 \%$ y RHA2 un $27 \%$. Trabajos previos con las mismas cenizas RHA1 y RHA2 en mezcla con $\mathrm{NaOH}$, orientados a producir soluciones de silicato de sodio, demostraron que la calidad de la fuente de sílice tiene un efecto significativo en la conversión de sílice a silicato (30). La sílice amorfa presenta gran reactividad en presencia de $\mathrm{NaOH}$, facilitando la formación del silicato de sodio a temperaturas bajas y tiempos de reacción menores a una hora, sin embargo la sílice cristalina presente también logra disolverse en alto grado aunque requiere mayor temperatura o tiempo de reacción; en cualquier caso los rendimientos fueron superiores al 90\% (30). Esto evidenció que tanto la sílice cristalina como la amorfa tienen la posibilidad de disolverse en la solución de hidróxido de sodio como consecuencia del alto contenido de álcalis en la mezcla, por lo cual para efectos de la dosificación en la preparación del silicato de sodio en el presente estudio se considero en ambos casos la sílice total presente en RHA1 y RHA2 (94\%).

Se fabricaron nueve sistemas, tres de referencia GBFSM1, FA-M1 y FA/GGBS-M1 utilizando como activante $\mathrm{SS}+\mathrm{NaOH}$ y seis sistemas donde se efectuó un reemplazo total del SS por RHA manteniendo las mismas dosificaciones, tal como se indica en la Tabla 2. El sistema binario FA/GBFS se preparó en proporción 50/50, la cual ha demostrado ser la óptima en este tipo de mezclas (31-33). La relación L/S, incluida en la misma Tabla, se calculó considerando L como el agua contenida en el silicato de sodio mas la adicional incorporada a la mezcla y S, como la fase solida contenida en el material cementante (FA y GGBS) mas la correspondiente contenida en la disolución del activante (SS, RHA y $\mathrm{NaOH})$. Esta relación se seleccionó estrictamente a partir de la trabajabilidad de cada sistema, de manera que todas las mezclan presentaran la misma fluidez. El tipo de curado en los tres sistemas se seleccionó tomando como referencia estudios previos (31-38), así para el sistema simple basado en FA el curado se realizo a $80^{\circ} \mathrm{C}$ durante 24 horas, mientras en el sistema de GBFS $100 \%$ y 50:50 FA/GBFS el curado se realizó en cámara húmeda (H.R. 95\%).

\subsection{Systems prepared and working conditions}

Three alkaline solutions were prepared from a mix of sodium hydroxide and sodium silicate (SS) or one of two types of rice husk ash (RHA1 or RHA2), respectively identified as M1, M2 and M3 (see Table 2). Solutions M2 and M3 were prepared 1 day prior to use and stored in closed containers at laboratory temperature. Earlier studies with mixes containing RHA1 or RHA2 and $\mathrm{NaOH}$, designed to yield sodium silicate solutions, showed that the quality of the silica source significantly affected the conversion of silica to silicate (30). Amorphous silica was highly reactive in the presence of $\mathrm{NaOH}$, favouring sodium silicate formation at low temperatures within the hour, whereas crystalline silica, which also exhibited good solubility, called for higher temperatures or longer reaction times. In both cases, solubility was higher than $90 \%$ (30). This proved that both crystalline and amorphous silica can dissolve in sodium hydroxide due to its high alkaline content. Consequently, in the present study sodium silicate dosage was calculated on the grounds of the total silica present in RHA1 and RHA2 (94\%).

Nine systems were prepared: three controls denominated GBFS-M1, FA-M1 and FA/GBFS-M1 using SS+NaOH as the activator, and six systems in which SS was wholly replaced by the same dose of RHA, as shown in Table 2. The binary system, FA/GBFS, was prepared with equal proportions of fly ash and blast furnace slag, which earlier studies showed to be optimal (31-33). The liquid/solid ratio $(L / S)$ given in the table was found taking $L$ to be the water in the sodium silicate plus the amount added to the mix, and $S$ to be the FA and GBFS plus the solid phase in the activating solution (SS or RHA and $\mathrm{NaOH}$ ). The $L / S$ ratio was determined strictly on the grounds of system workability in each case to ensure equal fluidity throughout. Curing conditions were based on the results of prior studies (31-38): the FA system was cured at $80{ }^{\circ} \mathrm{C}$ for 24 hours and the GBFS and 50:50 FA/GBFS systems in a humidity chamber (R.H. 95\%) at ambient temperature. 
Tabla 2 / Table 2

Disoluciones alcalinas y sistemas activados.

Alkaline solutions and activated systems.

\begin{tabular}{|c|c|c|c|c|c|c|}
\hline \multirow{2}{*}{$\begin{array}{l}\text { Material / } \\
\text { Materials }\end{array}$} & \multirow{2}{*}{$\begin{array}{l}\text { Disolución / } \\
\text { Disolution }\end{array}$} & \multirow{2}{*}{$\begin{array}{l}\text { Activador alcalino / } \\
\text { Alkaline activator }\end{array}$} & \multirow{2}{*}{$\begin{array}{l}\text { Modulo de solución / } \\
\text { Module solution, Ms } \\
\left(\mathrm{SiO}_{2} / \mathrm{Na}_{2} \mathrm{O}\right)\end{array}$} & \multicolumn{2}{|c|}{$\begin{array}{l}\text { Relaciones molares / } \\
\text { Molar relation }\end{array}$} & \multirow{2}{*}{$\begin{array}{c}\text { Relación L/S I } \\
\text { Ratio L/S }\end{array}$} \\
\hline & & & & $\mathrm{SiO}_{2} / \mathrm{Al}_{2} \mathrm{O}_{3}$ & $\mathrm{Na}_{2} \mathrm{O} / \mathrm{SiO}_{2}$ & \\
\hline \multirow{3}{*}{ GBFS } & M1 & $\mathrm{SS}+\mathrm{NaOH}$ & \multirow{3}{*}{1.20} & \multirow{3}{*}{5.1} & \multirow{3}{*}{0.66} & \multirow{3}{*}{0.30} \\
\hline & M2 & $\mathrm{RHA} 1+\mathrm{NaOH}$ & & & & \\
\hline & M3 & $\mathrm{RHA} 2+\mathrm{NaOH}$ & & & & \\
\hline \multirow{3}{*}{ FA } & M1 & $\mathrm{SS}+\mathrm{NaOH}$ & \multirow{3}{*}{0.49} & \multirow{3}{*}{4.0} & \multirow{3}{*}{0.25} & \multirow{3}{*}{0.15} \\
\hline & M2 & $\mathrm{RHA} 1+\mathrm{NaOH}$ & & & & \\
\hline & M3 & $\mathrm{RHA} 2+\mathrm{NaOH}$ & & & & \\
\hline \multirow{3}{*}{ FA/GBFS $50 / 50$} & M1 & $\mathrm{SS}+\mathrm{NaOH}$ & \multirow{3}{*}{0.19} & \multirow{3}{*}{4.0} & \multirow{3}{*}{0.25} & \multirow{3}{*}{0.23} \\
\hline & M2 & $\mathrm{RHA} 1+\mathrm{NaOH}$ & & & & \\
\hline & M3 & $\mathrm{RHA} 2+\mathrm{NaOH}$ & & & & \\
\hline
\end{tabular}

\subsection{Pruebas mecánicas y técnicas instrumentales de caracterización}

Para determinar la resistencia a la compresión del material activado se utilizaron probetas prismáticas de $1 \times 1 \times 6 \mathrm{~cm}$ y el ensayo se realizó a las edades de 1 y 7 días de curado.

Las características mineralógicas y microestructurales de los distintos sistemas activados se estudiaron por medio de técnicas de caracterización tales como, Difracción de Rayos X (DRX), Resonancia Magnética Nuclear (RMN), y Microscopia electrónica de barrido (MEB). El ensayo DRX se efectuó en un Difractómetro X'Pert MRD (PanAnalytical), se utilizó radiación $\mathrm{CuKa}_{1}$, el tubo de cobre trabajo a $45 \mathrm{Kv}$ y $40 \mathrm{~mA}$, y los datos se registraron en un rango de $8^{\circ}$ a $60^{\circ}(2 \theta)$ durante $30 \mathrm{~min}$. En los ensayos de ${ }^{29} \mathrm{Si}$ se utilizó un equipo BRUKER 400 Ultrashied AVANCE II, la frecuencia de resonancia usada fue de $79,5 \mathrm{MHz}$, con una velocidad de giro de $5 \mathrm{KHz}$, utilizando como estándar tetrametilsilano; el espectro fue obtenido después de

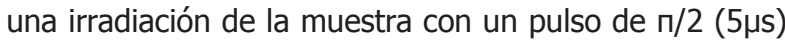
y tiempo entre acumulación de $5 \mathrm{~s}$. Por último la microestructura y los diferentes componentes de las pastas fueron observados en un microscopio electrónico de barrido marca JEOL, Ref: JSM-6490LV, alto vacío (3×10-6 torr), este equipo cuenta con un Detector INCAPentaFETx3 Marca OXFORD INTRUMENTS Modelo 7573; las muestras fueron recubiertas con oro por medio de deposición en una unidad de vacío Denton VacuumDesk IV.

\section{RESULTADOS Y DISCUSIÓN}

\subsection{Caracterización mecánica}

Las pastas activadas alcalinamente presentaron un comportamiento hidráulico similar a una pasta producida con cemento portland, a medida que pasa el tiempo se

\subsection{Mechanical tests and characterisation instruments}

The activated material was moulded into prismatic specimens measuring $1 \times 1 \times 6 \mathrm{~cm}$ and tested for compressive strength after 1 and 7 days.

System mineralogical and microstructural characteristics were studied using $X$-ray diffraction (XRD), nuclear magnetic resonance (NMR) and scanning electron microscopy (SEM). XRD analyses were conducted on a PanAnalytical X'Pert MRD diffractometer using CuKa ${ }_{1}$ radiation, a 45-kV, 40-mA copper $X$-ray tube, and 30-minute scans recorded in a $2 \theta$ range of 8 to $60^{\circ}$. The ${ }^{29} \mathrm{Si} N \mathrm{NMR}$ analyses were performed on a BRUKER 400 Ultrashield AVANCE II spectroscope under the following conditions: resonance frequency, $79.5 \mathrm{MHz}$; spinning speed, $5 \mathrm{kHz}$; internal standard, tetramethylsilane; sample radiation pulse, $\pi / 2$ (5 $\mu \mathrm{s})$; relaxation time, $5 \mathrm{~s}$. Lastly, paste microstructure and components were observed under a high vacuum ( $3 \times 10^{-6}$ torr) JEOL JSM-6490LV scanning electron microscope fitted with an OXFORD INSTRUMENTS 7573 INCAPentaFETX3 detector. The samples were gold-sputtered with a Denton VacuumDesk IV unit.

\section{RESULTS AND DISCUSSION}

\subsection{Mechanical Characterization}

The alkali-activated pastes exhibited hydraulic behaviour similar to portland cement pastes, with compressive strength rising with time irrespective of the activating 
incrementa en un orden apreciable la resistencia a la compresión, independiente del tipo de disolución activante utilizada (Figura 2). En general, los resultados de resistencia mecánica a compresión obtenidos en todas las mezclas a la edad de 7 días de curado, superan ampliamente la correspondiente de materiales producidos con base en cemento portland a la misma edad de curado (39-41); es de resaltar aquí también las resistencias a 1 día de curado de los sistemas activados, en especial las generadas por los sistemas de GBFS con valores entre 17 a $50 \mathrm{MPa}$ y los correspondientes de FA, con valores entre 35 y $67 \mathrm{MPa}$. A esta misma edad de curado, el sistema hibrido (FA) GBFS) reportó valores inferiores a $6 \mathrm{MPa}$, sin embargo su desempeño mecánico a la edad de 7 días de curado aumentó significativamente hasta alcanzar los $39 \mathrm{MPa}$. De los tres sistemas evaluados se destaca el de FA, lo cual es atribuible al curado térmico que contribuye a la ganancia resistente a edades cortas. En base en los resultados aquí expuestos, el curado debe ser considerado según el campo de aplicación del material y las características del material a utilizar. Así, en el caso de las escorias si se requieren resistencias mecánicas antes de 7 días es recomendable efectuar un curado térmico, teniendo en cuenta que después de esta edad la resistencia caerá ligeramente llegando a ser menor que la que se obtiene en el sistema curado a temperatura ambiente a la misma edad (38). solution used (Figure 2). As a rule, all the 7-day compressive strength values were substantially higher than found for portland cement materials at the same age (39-41). The 1-day data also showed considerable strength, particularly for the GBFS systems, with values of 17 to $50 \mathrm{MPa}$, and the FA samples, whose compressive strength ranged from 35 to $67 \mathrm{MPa}$. In this same age of curing the hybrid system (FA / GBFS) reported values lower than $6 \mathrm{MPa}$, however, its mechanical resistance to compression at 7 days of curing increased significantly reaching $39 \mathrm{MPa}$. Since thermal curing contributes to early age strength development, FA exhibited higher early age strength than the other systems studied. Based on these findings, curing should be designed in keeping with the intended application and the characteristics of the material in question. If high mechanical strength is needed in less than 7-day slag, for instance, thermal curing is recommended, bearing in mind that after that age, strength will dip to below the strength that would have been reached in 7-day material cured at ambient temperature (38).

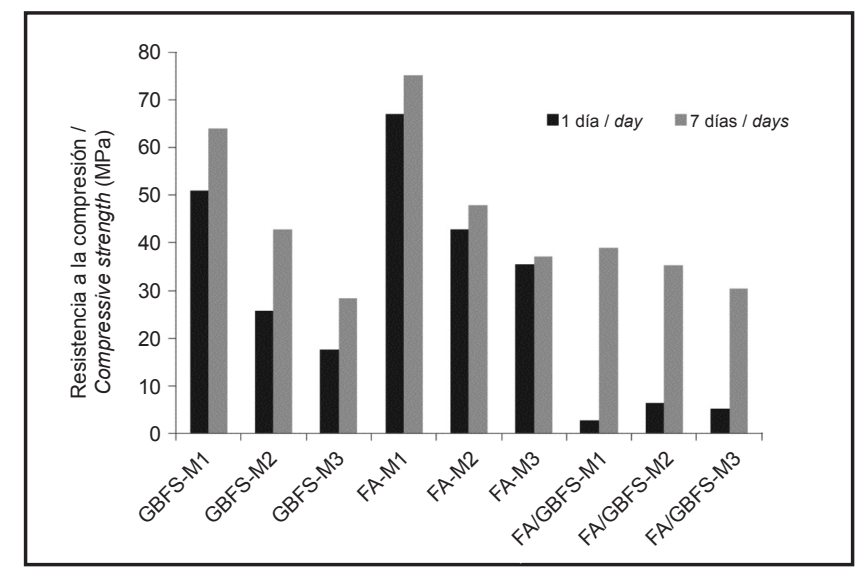

Figura 2. Resistencia a la compresión de los sistemas geopoliméricos. Figure 2. Compressive Strength of geopolymeric systems evaluated.

Respecto al efecto del tipo de disolución activante utilizada, en general, las pastas activadas con la solución alcalina M1 (empleando el silicato comercial) presentaron un comportamiento mecánico superior a aquellas activadas con las disoluciones M2 y M3, es decir donde el SS fue reemplazado en su totalidad por la ceniza de cascarilla de arroz. Al comparar los valores de resistencia mecánica a compresión de los sistemas activados con M2 y M3 se puede apreciar una diferencia de tan solo $10 \mathrm{MPa}$ entre sí, estos resultados permiten suponer que tanto la sílice amorfa como parte de la sílice cristalina presente en RHA2 pueden estar participando en el proceso de geopolimerización y formación de los productos de reacción.
The pastes activated with alkaline solution M1 (containing the commercial silicate) exhibited higher strength than the materials activated with solutions M2 and M3, in which the SS was fully replaced by rice husk ash. Since the difference in compressive strength between systems M2 and M3 was only $10 \mathrm{MPa}$, both the amorphous and part of the crystalline silica present in RHA2 might initiaIly be assumed to have participated in geopolymerisation and reaction product formation. 


\subsection{Caracterización mineralógica y microestructural de los sistemas activados alcalinamente}

Utilizando DRX se identificaron los principales productos de reacción cristalinos, tanto primarios como secundarios, de cada sistema evaluado. En la Figura 3 se muestran los difractogramas de las materias primas (GBFS y FA) y de algunas de las pastas obtenidas a los 7 días de curado. Cabe anotar, que una vez cumplido el tiempo de curado, las pastas fueron pulverizadas y sometidas a un proceso de congelamiento con acetona y etanol para detener el proceso de hidratación. En este estudio se incluyen los resultados comparativos de los tres sistemas activados alcalinamente al utilizar como disolución activante M1 (SS) y M3 (RHA proveniente del combustor).

\subsection{Mineralogical and microstructural characterisation of the alkali-activated systems}

The main primary and secondary crystalline reaction products were identified in each system with XRD. The diffractograms for the prime materials (GBFS and FA) and for some of the 7-day pastes are shown in Figure 3. After curing, the pastes were pulverised and submerged in acetone and ethanol to detain hydration. This study compared the findings for two of the three systems, alkali-activated with M1 (SS) and M3 (RHA2, the industrial by-product).
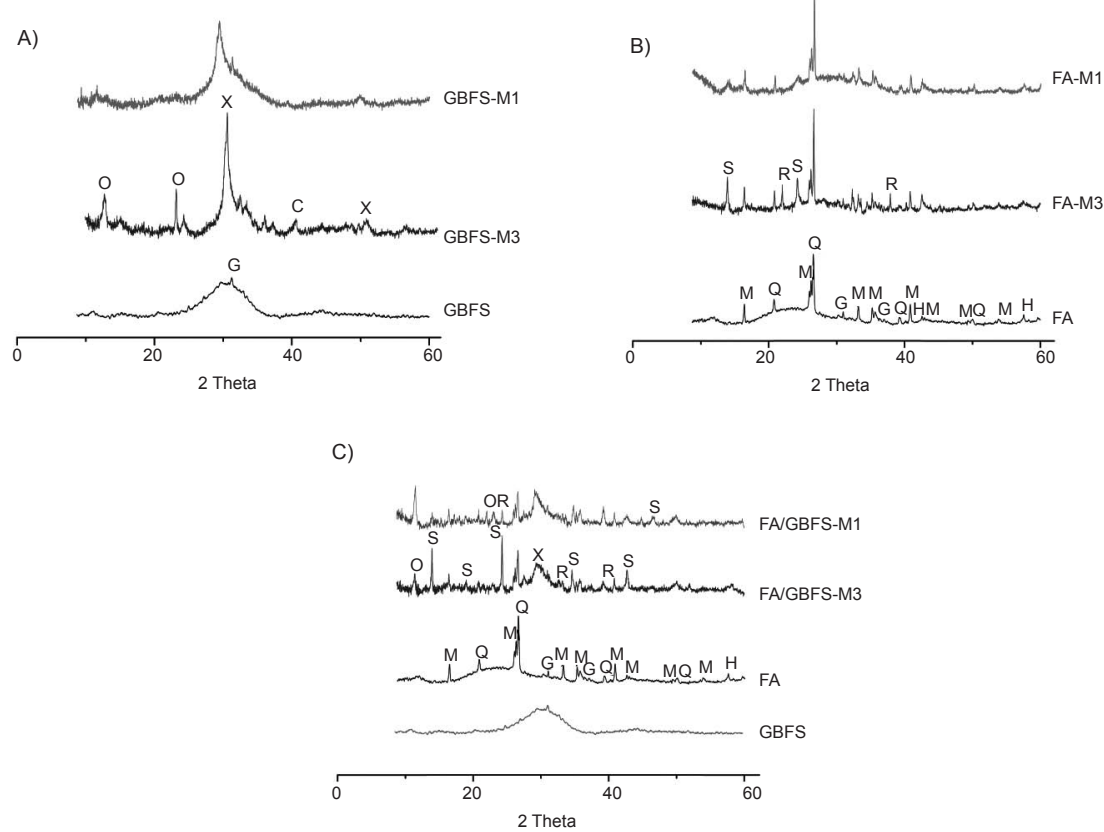

Figura 3. Patrón de difracción de rayos-X (DRX). a) GBFS-M1, GBFS-M3, b) FA-M1, FA-M3, c) FA/GBFS-M1, FA/GBFS-M3. X=C-S-H, $\mathrm{S}=$ hidroxisodalita, $\mathrm{M}=$ mullita, $\mathrm{R}=$ Hershelita o Na-chabacita, $\mathrm{C}=$ calcita, $\mathrm{O}=$ hidrotalcita, $\mathrm{G}=$ gelenita, $\mathrm{H}=$ hematita, $\mathrm{Q}=$ cuarzo. Figure 3. Pattern of X-ray diffraction (XRD). a) GBFS-M1, GBFS-M3, b) FA-M1, FA-M3, c) FA/GBFS-M1, FA/GBFS-M3. X=C-S-H, S = hydroxysodalite, $M=$ mullite, $R=$ Hershelita or Na-chabazite, $C=$ calcite, $O=$ hydrotalcite, $G=$ gehlenite, $H=$ hematite, $Q=$ quartz.

En los difractogramas de las pastas de escoria activada alcalinamente, tanto para el sistema GBFS-M1 como para GBFS-M3 (Figura 3a), se identificó como principal producto de reacción un silicoaluminato cálcico hidratado (tipo C-A-S-H) de baja cristalinidad, por las líneas de difracción en $2 \theta=29,56^{\circ}, 31,71^{\circ}$ y $49,89^{\circ}$ $\left(\mathrm{Ca}_{1.5} \mathrm{SiO}_{3.5} \cdot \mathrm{xH}_{2} \mathrm{O}\right)$, coincidiendo con resultados reportados por otros autores (42-44); aunque cabe anotar que en esta zona pueden presentarse solapamientos con fases propias de la escoria anhidra e incluso atribuibles a procesos de carbonatación. Adicional al gel C-A-S-H se detectó gelenita $\left(\mathrm{Ca}_{2} \mathrm{Al}_{2} \mathrm{SiO}_{7}\right)$ en $31,25^{\circ}(2 \theta)$,
The diffractograms for the slag pastes alkali-activated with $\mathrm{M} 1$ and $\mathrm{M} 3$ (Figure 3a) contained diffraction lines at $2 \theta=29.56^{\circ}, 31.71^{\circ}$ and $49.89^{\circ}\left(\mathrm{Ca}_{1.5} \mathrm{SiO}_{3.5} \times \mathrm{H}_{2} \mathrm{O}\right)$, which were identified with the main reaction product, low crystalline (C-A-S-H-like) calcium aluminosilicate hydrate. These findings concurred with the results reported by other authors (42-44). Nonetheless, phases present in the anhydrous slag or even attributable to carbonation may lead to overlapping in this region of the diffractogram. In addition to $C-A-S-H$ gel, the following phases were detected: gehlenite $\left(\mathrm{Ca}_{2} \mathrm{Al}_{2} \mathrm{SiO}_{7}\right)$ at $2 \theta=31.250^{\circ}$; calcite $\left(\mathrm{CaCO}_{3}\right)$ at $2 \theta=29.4$ and 39.40; and hydrotalcite 
calcita $\left(\mathrm{CaCO}_{3}\right)$ en 29,40 y 39,40 (20) e hidrotalcita $\left(\mathrm{Mg}_{6} \mathrm{Al}_{2}\left(\mathrm{CO}_{3}\right)(\mathrm{OH})_{16} \cdot 4\left(\mathrm{H}_{2} \mathrm{O}\right)\right)$ caracterizada por las reflexiones en $11,55^{\circ}$ y $22,90^{\circ}$ (20). Hay que destacar que el difractograma de la muestra GBFS-M3 muestra líneas de difracción más intensas que en la muestra GBFS-M1, indicando la formación de un mayor número de fases más cristalinas y un posible gel C-A-S-H con mayor orden estructural. Esto puede confirmarse en la observación por SEM incluida en la figura 4.
$\left(\mathrm{Mg}_{6} \mathrm{Al}{ }_{2}\left(\mathrm{CO}_{3}\right)(\mathrm{OH})_{16} \cdot 4\left(\mathrm{H}_{2} \mathrm{O}\right)\right)$, characterised by reflections at $2 \theta=11.55$ and $22.90^{\circ}$. The greater intensity of the lines for sample GBFS-M3 than for GBFS-M1 denoted a larger proportion of crystalline phases and the possible existence of a C-A-S-H gel with a higher range structural order. The latter was confirmed by the SEM micrograph reproduced in Figure 4.

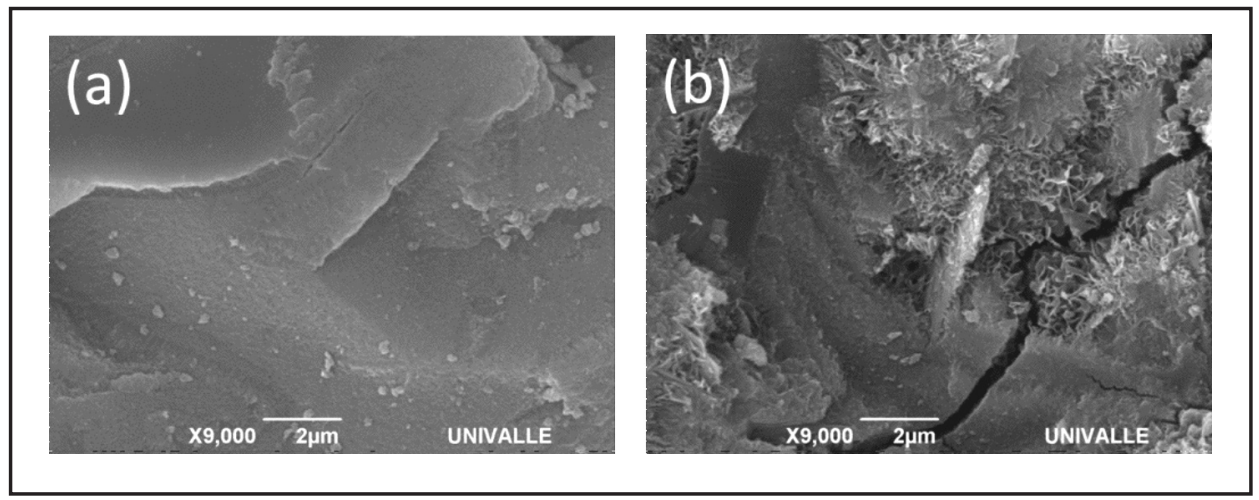

Figura 4. Microestructura MEB de sistemas a) GBFS-M1 y b) GBFS-M3.

Figure 4. Microstructure of alkali-activated systems (SEM) a) GBFS-M1 y b) GBFS-M3.

En los difractogramas de las pastas de ceniza volante activadas alcalinamente, FA-M1 y FA-M3 (Figura 3b), a diferencia de lo observado en los DRX de GBFS, no se identificaron fases tipo C-S-H o C-A-S-H. En esta clase de sistemas bajos en calcio el producto de reacción que se espera encontrar es un gel precursor zeolítico de tipo Na-aluminosilicato hidratado de tipo amorfo. Lógicamente, esta fase no se identificó por DRX, pero sí se detectó la presencia de zeolitas, lo cual parece indicar que parte del gel amorfo se pudo transformar en un producto de reacción secundario cristalino correspondiente a Na-Chabacita o también Ilamada Herschelita (R) $\left(\mathrm{NaAlSi}_{2} \mathrm{O}_{6} \cdot 3 \mathrm{H}_{2} \mathrm{O}\right)$ y sodalita o también llamada Hidroxisodalita (S) $\left(\mathrm{Na}_{4} \mathrm{Al}_{3} \mathrm{Si}_{3} \mathrm{O}_{12} \mathrm{OH}\right)$; estos compuestos han sido igualmente reportados por otros autores en sistemas de cenizas volantes activadas alcalinamente (45-48). La presencia de estas zeolitas se confirma mediante microscopia electrónica de barrido tal como puede observarse en la Figura 5. Cabe aclarar, que aunque ambos tipos de zeolitas se encuentran presentes en los dos sistemas, hay mayor formación de esta fase en el sistema FA-M3; otros compuestos cristalinos visualizados en estos difractogramas fueron: cuarzo (Q) y mullita (M), los cuales corresponden a componentes presentes originalmente en FA y en la ceniza de cascarilla que no han reaccionado en el medio alcalino.

Por último en los difractogramas de los sistemas híbridos FA/GBFS-M1 y FA/GBFS-M3 (Figura 3c) se observó la unión de los productos de reacción tanto de la escoria
Contrary to the patterns for GBFS, no C-S-H or C-A-S-Hlike phases were detected on the diffractograms for the alkali-activated fly ash pastes, FA-M1 and FA-M3 (Figure 3b). In these low calcium systems, the expected reaction product is a zeolite precursor gel, an amorphous Na-aluminosilicate hydrate. This phase was logically not detected by XRD. The fact that certain zeolites were, however, would appear to indicate that part of the amorphous gel may have converted into secondary crystalline reaction products such as Na-chabazite, also known as herschelite ( $\mathrm{R})\left(\mathrm{NaAlSi}_{2} \mathrm{O}_{6} \cdot 3 \mathrm{H}_{2} \mathrm{O}\right)$, and sodalite, otherwise denominated hydroxysodalite (S) $\left(\mathrm{Na}_{4} \mathrm{Al}_{3} \mathrm{Si}_{3} \mathrm{O}_{12} \mathrm{OH}\right)$. The presence of these compounds, also reported by other authors studying alkali-activated fly ash systems (45-48), was confirmed by scanning electron microscopy, as shown in Figure 5. While both types of zeolites were found in the two systems, they were more abundant in FA-M3. The other crystalline compounds detected on the diffractograms were quartz $(Q)$ and mullite $(M)$, components of the starting materials, FA and rice husk ash that failed to react in the alkaline medium.

The diffractograms for the two hybrid systems, $F A$ / GBFS-M1 and FA/GBFS-M3 (Figure 3c), revealed the coexistence of reaction products typical of both granulated 
vítrea de alto horno como de la ceniza volante. En ese caso se encontraron simultáneamente silico-aluminato de calcio hidratado (C-A-S-H) y la fase cristalina correspondiente a la transformación del precursor zeolítico en Hershelita (R) y Sodalita (S); así mismo se observaron las fases gelenita, calcita e hidrotalcita, cuarzo y mullita provenientes de los precursores GBFS y FA no reaccionados. blast furnace slag and fly ash activation. Calcium aluminosilicate hydrate (C-A-S-H) was detected, along with the intermediate crystalline phase in the conversion of the zeolite precursor to herschelite $(R)$ and sodalite $(S)$. Other phases identified included gehlenite, calcite and hydrotalcite, and the quartz and mullite present in the unreacted GBFS and FA.

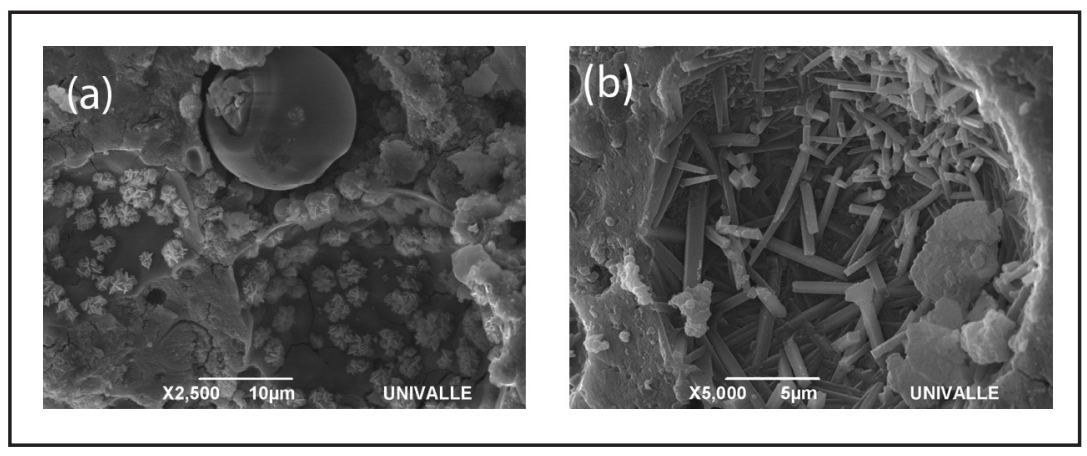

Figura 5. Microestructura MEB de sistemas a) y b) FA-M3.

Figure 5. Microstructure of alkali-activated systems (SEM) a) y b) FA-M3.

En base a estos resultados se infiere que el uso de la ceniza de cascarilla de arroz en los tres sistemas GBFS, FA, FA/GBFS, como remplazo total del silicato de sodio en la disolución activante no presenta mayor incidencia en el tipo de productos de reacción formados, aunque cabe resaltar que en los sistemas de FA activados con RHA2 (M3) se observa un incremento en la formación de compuestos zeolíticos. Este hecho se puede deber a la menor cantidad de sílice soluble en el medio en comparación con la cantidad de sílice soluble del silicato de sodio, capaz de reaccionar, en su totalidad, para formar productos de reacción tipo gel aluminosilicato de sodio hidratado. Esto parece indicar que la sílice cristalina presente en RHA2 participa solo parcialmente en la reacción geopolimérica.

En la Figura 6, se presentan los espectros de ${ }^{29} \mathrm{Si}$ RMNMAS de las materias primas anhídridas, y los diferentes sistemas evaluados (GBFS-M1 y GBFS-M3, FA-M1 y FA-M3, FA/GBFS-M1, FA/GBFS-M3). En cuanto a la materia prima anhídrida se observa, tanto el espectro de FA como en el de GBFS, una señal ancha, indicativa del carácter amorfo de estos materiales. En el espectro de FA se destacan señales ubicados entre -80 y -105 ppm correspondientes a la fase vítrea del material y entre -110 y -115 ppm pertenecientes a la fase cristalina, principalmente cuarzo identificado con las unidades $\mathrm{Q}^{4}(\mathrm{OAl})$ sí como también una señal ubicada en -90 ppm característica de la mullita Q33Al) (48). La escoria anhídrida por su parte está compuesta por unidades básicas de tetraedros de silicio individuales de tipo $\mathrm{Q}^{0} / \mathrm{Q}^{1}$ ubicados en $-69 \mathrm{ppm}$ y $-75 \mathrm{ppm}$, esta última señal no fue posible ubicarla por medio de deconvolución pero teniendo en cuenta el ancho del espectro es posible que se encuentre solapada.
What these findings show is that the total replacement of sodium silicate by rice husk ash in the activating solutions for the three systems, GBFS, FA and FA/GBFS, had no effect on the nature of the reaction products, although more zeolite compounds formed in the $F A$ systems activated with M3 (containing RHA2). The explanation for that observation may lie in the smaller amount of soluble silica in the medium compared to the amount present in sodium silicate, which can react in its entirety to form sodium aluminosilicate hydratelike reaction products. This finding would appear to suggest that the crystalline silica present in RHA2 participates partially in the geopolymer reaction.

Figure 6 shows the ${ }^{29} \mathrm{Si}$ MAS-NMR spectra for the anhydrous raw materials and the systems studied (GBFSM1 and GBFS-M3; FA-M1 and FA-M3; FA/GBFS-M1 and $F A / G B F S-M 3)$. The spectra for both the anhydrous $F A$ and GBFS exhibited a wide signal, denoting the amorphous nature of these materials. The prominent signals at -80 to -105 ppm on the FA spectrum were attributed to the vitreous phase of the material, the signals at -110 to $-115 \mathrm{ppm}$ to the crystalline phase, primarily to the quartz identified with $Q^{4}(O A /)$ units, and the signal located at $-90 \mathrm{ppm}$ to mullite $\left(Q^{3}(3 \mathrm{~A} /)\right)(48)$. The anhydrous slag, in turn, comprised $Q^{0} / Q^{1}$ type tetrahedral silicon monomers that generated signals at -69 and -75 ppm. Although the latter could not be positioned with deconvolution methods, its width would appear to suggest the existence of overlapping. 
Los sistemas GBFS-M1 y GBFS-M3 cuando son activados alcalinamente presentan un comportamiento similar. El proceso de transformación estructural, luego de la disolución y posterior polimerización, da lugar a la formación del gel silicoaluminato cálcico hidratado (tipo C-A-S-H) (12). Este es el producto primario de los sistemas alcalinos ricos en calcio, el cual se compone por dímeros de silicio de tipo $\mathrm{Q}^{2}(\mathrm{OAl})$ y $\mathrm{Q}^{2}(1 \mathrm{Al})$ que actúan como unidades de mitad de cadena ubicados en -85 y -82 ppm, respectivamente. En cuanto a las señales ubicadas entre -74 y -80 ppm podrían estar asociadas a unidades $\mathrm{Q}^{1}$ ubicadas en el final de cadena; y la señal en -88,6 ppm elucida el remplazo de átomos de silicio por átomos de aluminio en los tetraedros formando unidades de tipo $\mathrm{Q}^{3}(1 \mathrm{Al})(10,11)$. Las señales de $-69 \mathrm{ppm}$ y $-70 \mathrm{ppm}$ se asocian a escoria sin reaccionar $(9,49-51)$.

Por medio de esta técnica de RMN se evidenció la falta de disolución de algunas especies $\mathrm{Q}^{4}(0 \mathrm{Al})$ (-101,06 ppm) en el sistema FA-M3 (48), lo cual apoya los resultados menores de resistencia mecánica. Las unidades $\mathrm{Q}^{4}(\mathrm{OAl})$ del silicio reactivo, presentes en la ceniza volante tanto en FA-M1 como en FA-M3, una vez entran en contacto con el medio alcalino inician un proceso de polimerización y posterior condensación donde se incluye sustituciones isomórficas de los átomos de silicio por átomos de aluminio. En ambos espectros se visualizaron señales correspondientes a unidades $\mathrm{Q}^{4}(2 \mathrm{Al}), \mathrm{Q}^{4}(3 \mathrm{Al})$ y $\mathrm{Q}^{4}(4 \mathrm{Al})$ $(-96,-92,-86 \mathrm{ppm}$ respectivamente) con lo cual se infiere la sustitución del silicio por el aluminio en los tetraedros y la efectiva formación del producto de reacción en el sistema FA-M1 (46, 48-53). La unión de las especies de tipo $\mathrm{Q}^{4}(0 \mathrm{Al}, 1 \mathrm{Al}, 2 \mathrm{Al}, 3 \mathrm{Al}$ y $4 \mathrm{Al})$ forman cadenas poliméricas organizadas en una red de tipo $3 \mathrm{D}$, estructura característica del gel aluminosilicato sódico hidratado (NA-S-H); por otro lado concordando con el análisis de DRX se evidenció la formación de fases cristalinas, en ambos sistemas FA-M1 y FA-M3, correspondientes a zeolitas de tipo Hershelita identificadas en el espectro por las señales en -87 ppm para el sistema FA-M1 y en -87, -97 y -103 ppm en el sistema FA-M3 e hidroxisodalita, cuya señal se encuentra ubicada en -84 ppm para el caso del sistema FA-M3. Adicionalmente, se aprecian en el espectro de la pasta FA-M3 señales definidas y estrechas, las cuales indican que la proporción de material cristalino es mayor que en FA-M1, lo anterior confirma el resultado obtenido por medio de DRX.

En los sistemas híbridos FA/GBFS-M1 y FA/GBFS-M3 se evidenció la coexistencia de los dos tipos de productos de reacción originados a partir de la activación alcalina tanto de GBFS como de FA. En ambos espectros (Figura 6) se detectan señales $\mathrm{Q}^{0}, \mathrm{Q}^{1}, \mathrm{Q}^{2}$ y $\mathrm{Q}^{3}(1 \mathrm{Al})$ del gel silicoaluminato cálcico (tipo C-A-S-H) hidratado ubicadas entre -69 y -89 ppm, por otro lado el gel N-A-S-H lo definen las señales ubicadas entre -82 y -105 ppm, las cuales en
The alkali-activated GBFS-M1 and GBFS-M3 systems exhibited similar behaviour. In both, the structural transformation process, which is subsequent to dissolution and polymerisation, gave rise to a (C-A-S-H-like) calcium aluminosilicate hydrate. The primary product in calciumrich systems, this hydrate (12) comprises $Q^{2}(0 A)-$ and $Q^{2}(1 A /)-t y p e$ silicon dimers, mid-chain units that generate signals at -85 and -82 ppm, respectively. The signals located between -74 and -80 ppm might be attributed to end-of-chain $Q^{1}$ units, while the signal at $-88.6 \mathrm{ppm}$ was indicative of the replacement of silicon by aluminium atoms in the tetrahedra forming $Q^{3}(1 \mathrm{Al})$-type units (10, 11). The signals at -69 and $-70 \mathrm{ppm}$ were attributed to unreacted slag $(9,49-51)$.

NMR confirmed that some of the $Q^{4}(0 \mathrm{Al})(-101.06 \mathrm{ppm})$ species in the FA-M3 system failed to dissolve (48), a finding consistent with the lower mechanical strength values recorded for this system. Once the reactive silicon $Q^{4}(O A I)$ units, present in both FA-M1 and FA-M3 fly ash, came into contact with the alkaline medium, they underwent polymerisation and subsequent condensation, in which silicon atoms were isomorphically replaced by aluminium atoms. Both spectra exhibited signals generated by $Q^{4}(2 A l), Q^{4}(3 A l)$ and $Q^{4}(4 A l)$ units $(-96,-92,-86$ ppm respectively), denoting the replacement of silicon with aluminium in the tetrahedra and effective reaction product formation in System FA-M1 (46, 48-53). The bonding of $Q^{4}(O A l, 1 A l, 2 A l, 3 A l$ and $4 A l)$-type species formed polymer chains arranged in the three-dimensional network characteristic of sodium aluminosilicate hydrate $(\mathrm{N}-\mathrm{A}-\mathrm{S}-\mathrm{H}) \mathrm{gel}$. Moreover, crystalline phases were observed to form in both FA-M1 and FA-M3, corroborating the XRD findings. These phases were identified as herschelite-like zeolites, based on the signals at -87 ppm in the FA-M1 spectrum and at -87, -97 and -103 ppm in system FA-M3. Hydroxysodalite was also detected, with a signal at -84 ppm in the FA-M3 system. The spectrum for this system also exhibited narrow, highly resolved signals, an indication that the proportion of crystalline material was higher than in FA-M1, again corroborating the $X R D$ results.

The spectra for the FA/GBFS-M1 and FA/GBFS-M3 hybrid systems revealed the co-existence of the reaction products generated during the alkaline activation of both $G B F S$ and $F A$. Signals for $Q^{0}, Q^{1}, Q^{2}$ and $Q^{3}(1 A l)$ units in the (C-A-S-H-like) calcium aluminosilicate hydrate gel were detected on both spectra (Figure 6) at -60 to -89 ppm. The N-A-S-H gel, in turn, generated signals positioned at -82 to -105 ppm, which were fewer and 

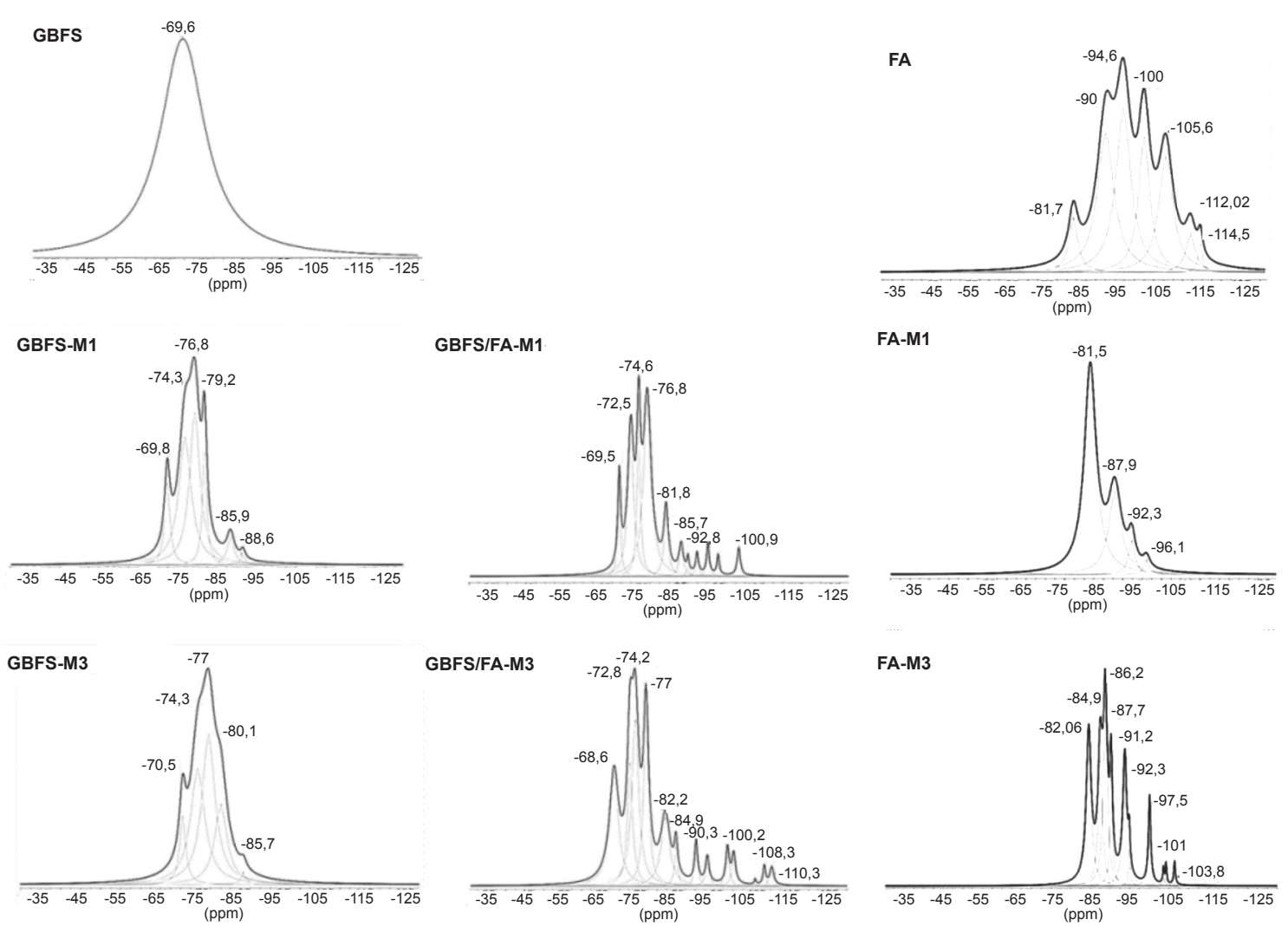

Figura 6. Espectros ${ }^{29}$ Si MAS-RMN. Sistemas activados alcalinamente. Figure 6. ${ }^{29}$ Si MAS-NMR spectra. Alkali activated systems.

comparación con las señales del gel C-A-S-H son pocas y con menor área; sin hacer un análisis cuantitativo solo cualitativo es posible inferir, a partir de los tamaños de las señales para cada producto de reacción una mayor formación de C-A-S-H que de N-A-S-H, cabe aclarar que en este rango de señales ya mencionadas hay solapamiento del gel C-A-S-H. En cuanto a la formación de productos cristalinos o material sin reaccionar se detectó, al igual que en el sistema FA 100\%, presencia de hidroxisodalita, hershelita; asimismo unidades $\mathrm{Q}^{4}$ (OAl) identificadas como fase cristalina (cuarzo) provenientes de FA no reaccionada o de la ceniza de cascarilla utilizada (RHA2) (48).

\section{CONCLUSIONES}

Los resultados aquí presentados evidencian que la ceniza de cascarilla de arroz, independiente de su contenido en sílice amorfa, puede ser utilizada como fuente de sílice en un sistema geopolimérico remplazando totalmente el silicato de sodio comercial. Esto evidencia la posibilidad de utilización del subproducto industrial proveniente del combustor, con un reducido nivel de sílice amorfa, e incrementa así su valor agregado a la par que contribuye a la mayor sostenibilidad ambiental del producto final. less intense than the $C-A-S-H$ gel signals. A qualitative analysis, based merely on the visible size of the signals for each reaction product, revealed that more $C-A-S-H$ than $N-A-S-H$ gel was formed, although overlapping occurred in the range associated with the former. As in the $100 \%$ FA system, the crystalline phases formed or present in the unreacted material included hydroxysodalite, herschelite and $Q^{4}(O A I)$ units, the third associated with the quartz present in the unreacted FA or the rice husk ash (RHA2) used (48).

\section{CONCLUSIONS}

The findings described here show that rice husk ash, irrespective of its amorphous silica content, can be used as the sole source of additional silica in geopolymer systems, completely replacing commercial sodium. This endorses the possibility of applying a combustion by-product with low amorphous silica content to alkali-activated binder production, contributing to both the valorisation of the waste and the environmental sustainability of the end product. 
Adicionalmente se comprueba que es posible obtener a partir de FA, GBFS o sistemas híbridos FA/GBFS materiales activados alcalinamente con resistencias mecánicas a edades tempranas (7 días) del orden de hasta $75 \mathrm{MPa}$ utilizando como activador silicato de sodio, y de hasta $42 \mathrm{MPa}$ al reemplazar en su totalidad el silicato por una ceniza de cascarilla de arroz. Estos valores superan ampliamente los correspondientes de materiales producidos con base exclusivamente en cemento portland a la misma edad de curado. Cabe anotar, que de los sistemas estudiados solo el sistema basado en FA $100 \%$ requiere curado térmico.

Finalmente, este estudio corrobora que tanto la sílice amorfa como parte de la sílice cristalina presente en la ceniza de cascarilla de arroz tienen la posibilidad de participar en el proceso de activación alcalina, siempre y cuando las condiciones de alcalinidad sean adecuadamente ajustadas.

\section{AGRADECIMIENTOS}

Las autoras expresan su agradecimiento al Departamento Administrativo de Ciencia y Tecnología de Colombia, Colciencias (Proyecto GEOCERAM), la Universidad del Valle (GMC), el Centro de Excelencia de Nuevos Materiales y al Instituto de Ciencias de la Construcción Eduardo Torroja de España por el apoyo en la ejecución del presente trabajo, y a la doctora Ana Fernández por facilitar la ceniza volante utilizada. También agradecen al MINECO por la subvención al proyecto BIA2010.15516.
The study also shows that alkali-activated materials can be obtained from FA, GBFS or hybrid FA/GBFS systems with 7-day strengths of up to $75 \mathrm{MPa}$ when sodium silicate is used in the activator, and up to $42 \mathrm{MPa}$ when that compound is wholly replaced by rice husk ash. These values are substantially higher than found for ordinary portland cement pastes of the same age. Of the systems studied, only the $100 \%$ FA required thermal curing.

This study confirms that both the amorphous silica and part of the crystalline silica present in rice husk ash may participate in alkaline activation, providing the alkalinity is suitably adjusted.

\section{BIBLIOGRAFÍA / BIBLIOGRAPHY}

(1) Davidovits, J.: Geopolymer chemistry and applications, pp. 3-12, Institut Geopolymere, Saint Quentin (2008).

(2) Pacheco-Torgal, F.; Castro-Gomes, J.; Jalali, S.: "Alkali-activated binders: A review Part 1. Historical background, terminology, reaction mechanisms and hydration products", Constr. and Build. Mater., vol. 22, nº 7 (2008), pp. 1305-1314. http://dx.doi.org/10.1016/j.conbuildmat.2007.10.015

(3) Cioffi, R.; Maffucci, L.; Santoro L.: "Optimization of geopolymer synthesis by calcination and polycondensation of a kaolinitic residue", Resources, Conservation and Recycling, vol. 40, n 1 (2003), pp. 27-38. http://dx.doi.org/10.1016/S0921-3449(03)00023-5

(4) Van Deventer, J.S.J.; Provis, J.L.; Duxson, P.: "Technical and commercial progress in the adoption of geopolymer cement", Minerals Engineering, vol. 29 (2012), pp. 89-104. http://dx.doi.org/10.1016/j.mineng.2011.09.009

(5) Komnitsas. K.; Zaharaki, D.: "Geopolymerisation: A review and prospects for the minerals industry", Minerals Engineering, vol. $20 \mathrm{n}^{\circ} 14$ (2007), pp. 1261-1277.

http://dx.doi.org/10.1016/j.mineng.2007.07.011

(6) Zheng, L.; Wang, C.; Wang, W.; Shi, Y.; Gao, X.: "Immobilization of MSWI fly ash through geopolymerization: Effects of water-wash", Waste Management, vol. 31, n² (2011), pp. 311-317. http://dx.doi.org/10.1016/j.wasman.2010.05.015

(7) Xu, H.; Van Deventer, J.S.J.: "The geopolymerisation of alumino-silicate minerals". Int. J. Miner. Process.; vol. 59, n 3 (2000), pp. $247-266$. http://dx.doi.org/10.1016/S0301-7516(99)00074-5

(8) García-Lodeiro, I.; Fernández-Jiménez, A.; Palomo, A.; Macphee, D.E.: "Effect of Calcium Additions on N-A-S-H Cementitious Gels", J. Am. Ceram. Soc., vol. 93, n० 7 (2010), pp. 1934-1940.

(9) Puertas, F.; Palacios, M.; Manzano, H.; Dolado, J.S.; Rico, A.; Rodríguez, J.: "A model for the C-A-S-H gel formed in alkali-activated slag cements", Journal of the European Ceramic Society, vol. 31, n 2 (2011), pp. 2043-2056. http://dx.doi.org/10.1016/j.jeurceramsoc.2011.04.036 
(10) Fernández-Jiménez, A.; Puertas, F.; Sobrados, I.; Sanz, J.: "Structure of Calcium Silicate Hydrates Formed in Alkaline-Activated Slag: Influence of the Type of Alkaline Activator", J. Am. Ceram. Soc., vol. 86, n 8 (2003), pp. 1389-1394. http://dx.doi.org/10.1111/j.1151-2916.2003. tb03481.x

(11) Puertas, F.; Fernández-Jiménez, A.; Blanco-Varela, M.T.: "Pore solution in alkali-activated slag cement pastes. Relation to the composition and structure of calcium silicate hydrate", Cem. Concr. Res., vol. 34, n 1 (2004), pp. 139-148. http://dx.doi.org/10.1016/S00088846(03)00254-0

(12) Bernal, S.A.; Mejía de Gutierrez, R.; Ruiz, F.; Quiñones, H.; Provis, J.L. "High-temperature performance of mortars and concretes base don alkali-activated slag/metakaolin blends", Mater Construcc, vol. 62, no 308 (2012), pp. 471-488. doi: 10.3989/mc.2012.0712.

(13) Sánchez, R.; Palacios, M.; Puertas, F.: "Cementos petroleros con adición de escoria de horno alto. Características y propiedades", Mater. Construcc., vol. 61, n 302 (2011), pp. 185-211. doi: 10.3989/mc.2010.54110.

(14) Duxson, P.; Fernández-Jiménez, A; Provis, J. L; Lukey, G. C.; Palomo, A; Van Deventer, J.S.J.: "Geopolymer Technology: The Current State of the Art", J. Mater. Sci, vol. 42 (2007), pp. 2917-2933. http://dx.doi.org/10.1007/s10853-006-0637-z

(15) Davidovits, J.: "30 Years of Successes and Failures in Geopolymer Applications. Market Trends and Potential Breakthroughs", Geopolymer 2002 Conference, Melbourne, Australia, October 28-29 (2002).

(16) Konstantinos, K. A.: "Potential of geopolymer technology towards green buildings and sustainable cities", Procedia Engineering, vol. 21 (2011), pp. 1023-1032. http://dx.doi.org/10.1016/j.proeng.2011.11.2108

(17) Juenger, M.C.G.; Winnefeld, F.; Provis, J.L.; Ideker, J.H.: "Advances in alternative cementitious binders", Cem. Concr. Res., vol. 41, $\mathrm{n}^{\circ}$ 12 (2011), pp. 1232-1243. http://dx.doi.org/10.1016/j.cemconres.2010.11.012

(18) Shi, C.; Fernández-Jiménez, A.; Palomo, A.: "New cements for the 21st century: The pursuit of an alternative to Portland cement", Cem. Concr. Res., vol. 41, n7 (2011), pp. 750-763. http://dx.doi.org/10.1016/j.cemconres.2011.03.016

(19) Van Deventer, J.S.J.; Provis, J.L.; Duxson, P.; Brice, D.G.: "Chemical research and climate change as drivers in the commercial adoption of alkali activated materials", Waste Biomass Valor, vol. 1, n 1 (2010), pp. 145-155. http://dx.doi.org/10.1007/s12649-010-9015-9

(20) Fernández-Jiménez, A.; Palomo, A.: "Composition and microstructure of alkali activated fly ash binder: Effect of the activator", Cem. Concr. Res., vol. 35, nº 10 (2005), pp.1984-1992. http://dx.doi.org/10.1016/j.cemconres.2005.03.003

(21) Fernandez-Jiménez, A.; Palomo, A.; Criado, M.: "Alkali activated fly ash binders. A comparative study between sodium and potassium activators". Mater. Construcc., vol. 56, n²81 (2006), pp. 52-65.

(22) Oh, J. E.; Monteiro, P.J.M.; Jun, S.S.; Choi, S.; Clark, S. M.: "The evolution of strength and crystalline phases for alkali-activated ground blast furnace slag and fly ash-based geopolymers", Cem. Concr. Res., vol. 40, n 2 (2010), pp. 189-196. http://dx.doi.org/10.1016/j. cemconres.2009.10.010

(23) Panias, D.; Giannopoulou, I.P.; Perraki, T.: "Effect of synthesis parameters on the mechanical properties of fly ash-based geopolymers", Colloids and Surfaces A: Physicochem. Eng. Aspects, vol. 301, n 1-3 (2007), pp. 246-254. http://dx.doi.org/10.1016/j.colsurfa.2006.12.064

(24) Řezník, B.; Frýbortová, I.; Rovnaníková, P.: "Properties of alkali-activated fly ash with respect to the silicate modulus of activator", $4^{\text {th }}$ International Conference Non-traditional Cement \& Concrete (2011), pp. 139-147.

(25) Provis, J.L.; Yong, C.Z.; Duxson P.; Van Deventer, J.S.J.: "Correlating mechanical and thermal properties of sodium silicate-fly ash geopolymers", Colloids and Surfaces A: Physicochem. Eng. Aspects, vol. 336, n 1-3 (2009), pp. 57-63. http://dx.doi.org/10.1016/j.colsurfa.2008.11.019

(26) Nazari, A.; Bagheri, A.; Riahi, S.: "Properties of geopolymer with seeded fly ash and rice husk bark ash", Materials Science and Engineering $A$, vol. 528, n 24 (2011), pp. 7395-7401. http://dx.doi.org/10.1016/j.msea.2011.06.027

(27) Mehta, P. K.: Belgium Patent 802909, July 1973.

(28) Palacios, M.; Puertas, F.: "Effectiveness of mixing time on hardened properties of waterglass-activated slag pastes and mortars", $A C I$ Materials Journal, vol. 108, n 1 (2011), pp. 73-78.

(29) Fernández-Jiménez, A.; Palomo, A.: "Characterisation of fly ash. Potencial reactivity as alkaline cements", Fuel, vol. 82, n 18 (2003), pp. 2259-2265. http://dx.doi.org/10.1016/S0016-2361(03)00194-7

(30) Bejarano, J.; Garzón, C.; Mejía de Gutiérrez, R.; Delvasto, S.; Gordillo, M.: "Obtención de soluciones de silicato de sodio a partir de cenizas de cascarilla de arroz", II Simposio Aprovechamiento de residuos agro-industriales como fuente sostenible de materiales de construcción. Valencia, 8 y 9 de noviembre de 2010 (pp. 409-418).

(31) Puertas, F.; Martínez-Ramírez, S.; Alonso, S.; Vázquez, T.: "Alkali-activated fly ash/slag cement Strength behaviour and hydration products", Cem. Concr. Res., vol. 30, nº 10 (2000), pp. 1625-1632. http://dx.doi.org/10.1016/S0008-8846(00)00298-2

(32) Puertas, F.; Fernández-Jiménez, A.: "Mineralogical and microstructural characterization of alkali-activated fly ash/slag pastes", Cem. Concr. Comp., vol. 25, n 3 (2003), pp. 287-292. http://dx.doi.org/10.1016/S0958-9465(02)00059-8

(33) Kumar, S.; Kumar, R.; Mehrotra, S. P.: "Influence of granulated blast furnace slag on the reaction, structure and properties of fly ash based geopolymer", J. Mater. Sci., vol. 45, n 3 (2010), pp. 607-615. http://dx.doi.org/10.1007/s10853-009-3934-5

(34) Temuujin, J.; Williams, R.P.; Van Riessen, A.: "Effect of mechanical activation of fly ash on the properties of geopolymer cured at ambient temperature", Journal of Materials Processing Technology, vol. 209, n 12-13 (2009), pp. 5276-5280.

http://dx.doi.org/10.1016/j.jmatprotec.2009.03.016 
(35) Temuujin, J; Van Riessen, A; Williams, R.: "Influence of calcium compounds on the mechanical properties of fly ash geopolymer pastes", Journal of Hazardous Materials, vol. 167, nº1-3 (2009), pp. 82-88. http://dx.doi.org/10.1016/j.jhazmat.2008.12.121 PMid:19201089

(36) Izquierdo, M.; Querol, X.; Phillipart, C.; Antenucci, D.; Towler, M.: "The role of open and closed curing conditions on the leaching properties of fly ash-slag-based geopolymers", Journal of Hazardous Materials, vol. 176, n¹-3 (2010), pp. 623-628. http://dx.doi.org/10.1016/j. jhazmat.2009.11.075 PMid:20005626

(37) Izquierdo, M.; Querol, X.; Davidovits, J.; Antenucci, D.; Nugteren, H.; Fernández-Pereira, C.: "Coal fly ash-slag-based geopolymers: Microstructure and metal leaching", Journal of Hazardous Materials, vol. 166, n¹ (2009), pp. 561-566. http://dx.doi.org/10.1016/j.jhazmat.2008.11.063 PMid:19118943

(38) Fernández-Jiménez, A.; Palomo, J.G.; Puertas, F.: "Alkali-activated slag mortars. Mechanical strength behavior". Cem. Concr. Res., vol. 29, n¹ (1999), pp. 1313-1321. http://dx.doi.org/10.1016/S0008-8846(99)00154-4

(39) Reza, M.; Karbalaie, M.: "An experimental study on compressive strength of concrete containing crumb rubber", International Journal of Civil \& Environmental Engineering, vol. 11, nº 03 (2011), pp. 24-28.

(40) Huang, B.; Shu, X.; Li, G.: "Laboratory investigation of portland cement concrete containing recycled asphalt pavements", Cem. Concr. Res., vol. 35, n 10 (2005), pp. 2008-2013. http://dx.doi.org/10.1016/j.cemconres.2005.05.002

(41) Wu, X.; Zhu, H.; Hou, X.; Li, H.: "Study on steel slag and fly ash composite Portland cement", Cem. Concr. Res., vol. 29, n 7 (1999), pp. 1103-1106.

(42) Bernal, S.; Herfort, D.; Skibsted, J.: "Hybrid binders based on alkali sulfate-activated Portland clinker and metacaolín", XIII ICCC International congress on the chemistry of cement, Madrid, 3-8 July, 2011.

(43) Lecomte, I.; Henrist, C.; Liégeois, M.; Maseri, F.; Rulmont, A.; Cloots, R.: "(Micro)-structural comparison between geopolymers, alkaliactivated slag cement and Portland cement", Journal of the European Ceramic Society, vol. 26, n 16 (2006), pp. 3789-3797. http://dx.doi. org/10.1016/j.jeurceramsoc.2005.12.021

(44) García-Lodeiro, I.; Palomo, A.; Fernández-Jiménez, A.; Macphee D.E.: "Compatibility studies between N-A-S-H and C-A-S-H gels. Study in the ternary diagram Na2O-CaO-Al2O3-SiO2-H2O", Cem. Concr. Res., vol. 41, no 9 (2011), pp. 923-931. http://dx.doi.org/10.1016/j. cemconres.2011.05.006

(45) Kovalchuk, G.; Fernández-Jiménez, A.; Palomo, A.: "Alkali-activated fly ash. Relationship between mechanical strength gains and initial ash chemistry", Mater. Construcc., vol. 58, n²91 (2008), pp. 35-52.

(46) Fernández-Jiménez, A.; Palomo, A.: "New Cementitious Materials Based on Alkali-Activated Fly Ash: Performance at High Temperatures", J. Am. Ceram. Soc.; vol. 91, nº10 (2008), pp. 3308-3314. http://dx.doi.org/10.1111/j.1551-2916.2008.02625.x

(47) Criado, M.; Fernández-Jiménez, A.; De La Torre, A.G.; Aranda, M.A.G.; Palomo, A.: "An XRD study of the effect of the SiO2/Na2O ratio on the alkali activation of fly ash", Cem. Concr. Res., vol. 37, n' 5 (2007), pp. 671-679. http://dx.doi.org/10.1016/j.cemconres.2007.01.013 (48) Criado, M.; Fernández-Jiménez, A.; Palomo, A.; Sobrados, I.; Sanz, J.: "Effect of the $\mathrm{SiO}_{2} / \mathrm{Na}_{2} \mathrm{O}$ ratio on the alkali activation of fly ash. Part II: 29Si MAS-NMR Survey", Microporous and Mesoporous Materials, vol.109, n 1-3 (2008), pp. 525-534. http://dx.doi.org/10.1016/j. micromeso.2007.05.062

(49) Palacios, M.; Puertas, F.: "Effect of Carbonation on Alkali-Activated Slag Paste", J. Am. Ceram. Soc, vol. 89 n 10 (2006), pp. $3211-3221$. http://dx.doi.org/10.1111/j.1551-2916.2006.01214.x

(50) Schneider, J. A.; Cincotto, M.; Panepucci, H.: "29Si and 27Al high-resolution NMR characterization of calcium silicate hydrate phases in activated blast-furnace slag pastes", Cem. Concr. Res., vol. 31, n 7 (2001), pp. 993-1000. http://dx.doi.org/10.1016/S0008-8846(01)00530-0 (51) Wang, S. D.; Scrivener, K.L.: "Si-29 and Al-27 NMR study of alkali-activated slag", Cem. Concr. Res., vol. 33, n 5 (2003), pp. $769-774$. http://dx.doi.org/10.1016/S0008-8846(02)01044-X

(52) Criado, M.; Fernández-Jiménez, A.; Palomo, A.: "Alkali activation of fly ash. Part III: Effect of curing conditions on reaction and its graphical description", Fuel, vol. 89, n¹1 (2010), pp. 3185-3192. http://dx.doi.org/10.1016/j.fuel.2010.03.051

(53) Fernández-Jiménez, A.; Monzó, M.; Vicent, M.; Barba, A.; Palomo, A.: "Alkaline activation of metakaolin-fly ash mixtures: Obtain of Zeoceramics and Zeocements", Microporous and Mesoporous Materials, vol. 108, n 1-3 (2008), pp. 41-49. http://dx.doi.org/10.1016/j. micromeso.2007.03.024 